\title{
Guided Biofilm Therapy
}

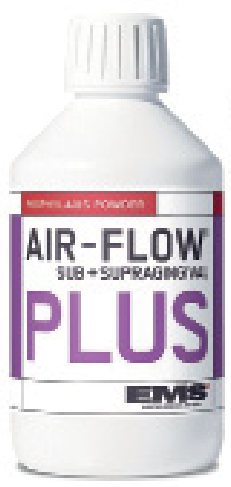

In einer Gemeinschaftsarbeit mit dem Prophylaxespezialisten $\mathrm{Dr}$. Klaus-Dieter Bastendorf hat EMS eine neue, systemische Vorgehensweise für die professionelle Zahnprophylaxe entwickelt. Gestützt auf zahlreichen wissenschaftlichen Studien stehen im Zentrum der sogenannten „Guided Biofilm Therapy" die State-of-the-Art-Technologien „Air-Flow“ und „Piezon No Pain“ zur Entfernung von harten sowie weichen Belägen und eine veränderte Reihenfolge des Behandlungsablaufs. Nach diesen Erkenntnissen ist die Entfernung von unterschiedlichen Zahnbelägen mithilfe von Hand- und Schall- bzw. Ultraschallinstrumenten zu Beginn der Behandlung überholt. Der Prozess gliedert sich nach der folgenden Reihenfolge: Diagnose, Entfernung aller weichen Ablagerungen auf Zahn-Hartsubstanz mit Air-Flow, Entfernung aller harten Ablagerungen auf ZahnHartsubstanz mit Piezon No Pain, Kontrolle, Abschluss, ggf. mit Air-Polishing; che- misch unterstützende Plaquekontrolle und Kontrolle durch den Zahnarzt und Recall-Terminvereinbarung.

Im Rahmen der „Guided Biofilm Therapy“ werden gleich im 1 . Schritt alle weichen Ablagerungen und der Biofilm auf Zahnhartsubstanz mit der Original Air-FlowMethode in Kombination mit dem AirFlow Pulver PLUS auf Erythritolbasis entfernt. Die besondere Wirkung des AirFlow Pulver PLUS ist auf den Inhaltsstoff Erythritol und die extrafeine Körnung von nur 14 Mikron zurückzuführen. Bei Erythritol handelt es sich um einen bewährten Zuckerersatzstoff. Die besonders kleinen Erythritolkörner ermöglichen einen sehr dichten Pulverstrahl mit hoher supra- und subgingivaler Reinigungswirkung. Dadurch wird der Biofilm in den kritischen Regionen wie Zahnfleischsaum, Zahnzwischenraum, Fissuren und subgingival mit dem Air-Flow Pulver PLUS erreicht und sicher entfernt. Der Patient empfindet dies als wohltuend. Erst im Anschluss daran entfernt man die nun deutlich sichtbaren harten Beläge (Piezon NO Pain).

Nach einer Pressemitteilung der EMS Electro Medical Systems GmbH, München www.byebyebiofilm.com 